\title{
A Space-Mapping Approach to Microwave Device Modeling Exploiting Fuzzy Systems
}

\author{
Slawomir Koziel, Senior Member, IEEE, and John W. Bandler, Life Fellow, IEEE
}

\begin{abstract}
We present a novel surrogate modeling methodology based on a combination of space mapping and fuzzy systems. Fine model data, the so-called base set, is assumed available in the region of interest. Although we do not assume any particular location of the base points, it is preferable that they form a uniform mesh. The standard space-mapping surrogate is established using available fine model data. The fuzzy system is then set up to interpolate the differences between the space-mapping surrogate and the fine model at all base points. Our new methodology offers significant advantages with respect to some of the previous space-mapping approaches to modeling, which are: 1) it handles any base set and 2) the number of space-mapping parameters does not limit the accuracy of the surrogate. Moreover, it exhibits comparable or better accuracy than the recently published modeling technique utilizing space mapping and radial basis functions. We also consider a hierarchical fuzzy space-mapping modeling, which relies on a fuzzy interpolation of space-mapping parameters and subsequent fuzzy interpolation of the residuals between the fine and surrogate model. Examples demonstrate the robustness of our approach and give a comparison with other space-mapping-based modeling techniques.
\end{abstract}

Index Terms-Computer-aided design (CAD), electromagnetic (EM) modeling, fuzzy systems, microwave circuits, space mapping, surrogate modeling.

\section{INTRODUCTION}

$\mathbf{S}$ TATISTICAL analysis and yield optimization are crucial to manufacturability-driven designs in a time-to-market development environment and demand fast accurate device and component models. Full-wave electromagnetic (EM) simulations of microwave structures offer accuracy at the cost of CPU effort. High CPU cost is undesirable from the point of view of direct statistical analysis and design. The space-mapping concept [1]-[8] addresses this issue.

Space mapping assumes the existence of "fine" and "coarse" models. The "fine" model may be a high fidelity CPU-intensive EM simulator, undesirable for direct statistical analysis and design. The "coarse" model can be a simplified representation such as an equivalent circuit with empirical formulas. Space-mapping modeling [9]-[16] and neuro-space-mapping

Manuscript received May 25, 2007; revised September 13, 2007. This work was supported in part by the Natural Sciences and Engineering Research Council of Canada under Grant OGP0007239 and Grant STPGP336760, and by Bandler Corporation.

S. Koziel is with the Simulation Optimization Systems Research Laboratory, Department of Electrical and Computer Engineering, McMaster University, Hamilton, ON, Canada L8S 4K1 (e-mail: koziels@mcmaster.ca).

J. W. Bandler is with the Simulation Optimization Systems Research Laboratory, Department of Electrical and Computer Engineering, McMaster University, Hamilton, ON, Canada L8S 4K1, and also with Bandler Corporation, Dundas, ON, Canada L9H 5E7 (e-mail: bandler@mcmaster.ca).

Digital Object Identifier 10.1109/TMTT.2007.909605 modeling [17]-[19] exploit the speed of the coarse model and the accuracy of the fine model to develop fast accurate enhanced models (surrogates) valid over a wide range of parameter values. The main factor that distinguishes space mapping from many other surrogate-based modeling methodologies (e.g., [20]-[26]) is the use of physics-based coarse models, which allows good modeling accuracy with a small amount of fine model data.

The standard space-mapping modeling methodology [11], [12] is based on setting up the surrogate model using a small amount of fine-model data (usually, $2 n+1$ points, where $n$ is the number of design variables). Extraction of the model parameters is performed over the whole set of this data. This methodology is simple and gives reasonable accuracy, which, however, may not be sufficient for some applications. To improve modeling performance, additional fine model information needs to be involved. Unfortunately, this approach to space mapping is not able to effectively harness a large amount of data, i.e., increasing the number of base points does not help if the number of space-mapping parameters (model flexibility) remains unchanged [15].

Space-mapping modeling with variable weight coefficients [14], [15] is aimed at overcoming these limitations. It indeed provides much better accuracy than the standard method, however, at the expense of significant increase of the evaluation time, which is due to a separate parameter extraction required for each evaluation of the surrogate model. This limits potential applications of the method.

A recently published modeling technique utilizing space mapping and radial basis function interpolation [16] gives modeling accuracy comparable with [14] without compromising computational cost. Moreover, because of the fact that the surrogate is based on the underlying coarse model, modeling accuracy is substantially better than for radial basis function interpolation used directly. Unfortunately, the problem of determining interpolation coefficients may be ill conditioned and the method may be very sensitive to some control parameters.

In this paper, we present another approach that combines standard space-mapping modeling with fuzzy system interpolation. This technique has the same advantages as the methodology [16], however, it is simpler to implement. Moreover, in some cases, it allows us to improve modeling accuracy even further.

\section{FuzZy Space-Mapping Surrogate Modeling}

Let $\boldsymbol{R}_{f}: X_{f} \rightarrow R^{m}, X_{f} \subseteq R^{n}$, and $\boldsymbol{R}_{c}: X_{c} \rightarrow R^{m}, X_{c} \subseteq$ $R^{n}$ denote the fine and coarse model response vectors. For example, $\boldsymbol{R}_{f}(\boldsymbol{x})$ and $\boldsymbol{R}_{c}(\boldsymbol{x})$ may represent the magnitude of 
a transfer function at $m$ chosen frequencies. We denote by $X_{R} \subseteq X_{f}$ the region of interest in which we want an enhanced matching between the surrogate and fine model. We assume that $X_{R}$ is an $n$-dimensional interval in $R^{n}$ with the center at reference point $\boldsymbol{x}^{0}=\left[x_{0.1} \ldots x_{0 . n}\right]^{T} \in R^{n}$

$$
\begin{aligned}
X_{R}= & {\left[\boldsymbol{x}^{0}-\boldsymbol{\delta}, \boldsymbol{x}^{0}+\boldsymbol{\delta}\right] } \\
= & {\left[x_{0.1}-\delta_{1}, x_{0.1}+\delta_{1}\right] \times \cdots } \\
& \times\left[x_{0 . n}-\delta_{n}, x_{0 . n}+\delta_{n}\right]
\end{aligned}
$$

where $\delta=\left[\delta_{1} \ldots \delta_{n}\right]^{T}$ determines the size of $X_{R}$. We use $X_{R}\left(\boldsymbol{x}^{0}, \boldsymbol{\delta}\right)$ to denote the region of interest defined by $\boldsymbol{x}^{0}$ and $\boldsymbol{\delta}$. Suppose we have the base set $X_{B}=\left\{\boldsymbol{x}^{1}, \boldsymbol{x}^{2}, \ldots, \boldsymbol{x}^{N}\right\} \subset$ $X_{R}\left(\boldsymbol{x}^{0}, \boldsymbol{\delta}\right)$, where $N$ is the number of base points, such that the fine model response is known at all points $\boldsymbol{x}^{j}, j=1,2, \ldots, N$.

A generic surrogate model $\overline{\boldsymbol{R}}_{s}$ is defined as in [16]

$$
\overline{\boldsymbol{R}}_{s}(\boldsymbol{x}, A, B, c)=\boldsymbol{A} \cdot \boldsymbol{R}_{c}(B \cdot x+c) .
$$

Matrices $\boldsymbol{A}, \boldsymbol{B}$, and $\boldsymbol{c}$ are obtained by the parameter extraction

$$
(A, \boldsymbol{B}, \boldsymbol{c})=\arg \min _{(\alpha, \beta, \gamma)} \sum_{k=1}^{N}\left\|\boldsymbol{R}_{f}\left(\boldsymbol{x}^{k}\right)-\overline{\boldsymbol{R}}_{s}\left(\boldsymbol{x}^{k}, \boldsymbol{\alpha}, \boldsymbol{\beta}, \boldsymbol{\gamma}\right)\right\| .
$$

Apart from model (2) and (3), optional frequency scaling can be implemented that works in such a way that the coarse model is evaluated at a different frequency than the fine model using the transformation $\omega \rightarrow f_{0}+f_{1} \omega$ [11]. More general spacemapping models can be found, e.g., in [3] and [11].

Let us introduce the so-called characteristic distance $\lambda=$ $\lambda(\boldsymbol{\delta}, N)$ of the base set depending on the size of the region of interest and the number of base points, defined as

$$
\lambda(\boldsymbol{\delta}, N)=\frac{2}{n N^{1 / n}} \sum_{i=1}^{n} \delta_{i} .
$$

If the base points are uniformly distributed in $X_{R}, \lambda=$ $\lambda(\boldsymbol{\delta}, N)$ is just an average distance between neighboring points. We will use parameter $\lambda$ to characterize and compare different base sets $X_{B}$.

On top of the standard space-mapping surrogate, we use fuzzy interpolation of the difference between the fine model $\boldsymbol{R}_{f}$ and standard surrogate. Fuzzy systems have been successfully used in the microwave area by other authors (e.g., [27]-[29]). In this study, we use a fuzzy system with triangle membership functions and centroid defuzzification [30]. We assume that we have data pairs $\left(\boldsymbol{x}^{k}, \boldsymbol{R}^{k}\right)$, where $\boldsymbol{x}^{k} \in X_{B}$ and $\boldsymbol{R}^{k}=\boldsymbol{R}_{f}\left(\boldsymbol{x}^{k}\right)-$ $\overline{\boldsymbol{R}}_{s}\left(\boldsymbol{x}^{k}\right), k=1,2, \ldots, N$. Membership functions for the $i$ th variable are defined as shown in Fig. 1. Each interval $\left[x_{0 . i}-\right.$ $\left.\delta_{0 . i}, x_{0 . i}+\delta_{0 . i}\right], i=1,2, \ldots, n$, is divided into $K$ subintervals (fuzzy regions). The number $K$ corresponds to the number of base points $N$ and is given by the formula $K=\left\lfloor N^{1 / n}\right\rfloor-1$. In particular, if $X_{B}$ consists of base points uniformly distributed in the region of interest $X_{R}$, then $K+1$ is exactly the number of points of this uniform grid along any of the design variable axes. In general, $K$ is chosen in such a way that the number of

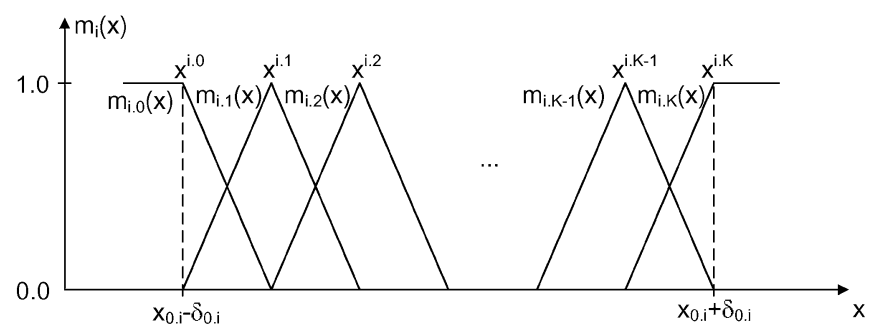

Fig. 1. Division of the input interval $\left[x_{0 . i}-\delta_{0 . i}, x_{0 . i}+\delta_{0 . i}\right]$ into fuzzy regions and the corresponding membership functions.

$n$-dimensional subintervals (and, consequently, the maximum number of rules) is not larger than the number of base points. The division of $\left[x_{0 . i}-\delta_{0 . i}, x_{0 . i}+\delta_{0 . i}\right]$ into $K$ subintervals creates $K+1$ values $x^{i . k}, k=0,1, \ldots, K$. In the case of a uniform base set, points $\boldsymbol{x}^{\boldsymbol{q}}=\left[x^{1 . q_{1}} \ldots x^{n \cdot q_{n}}\right]^{T}, \boldsymbol{q} \in\{0,1, \ldots, K\}^{n}$ coincide with the base points. Value $x^{i . k}$ corresponds to the fuzzy region $\left[x^{i . k-1}, x^{i . k+1}\right]$ for $k=1, \ldots, K-1\left(\left[x^{i .0}, x^{i .1}\right]\right.$ for $k=0$, and $\left[x^{i . K-1}, x^{i . K}\right]$ for $\left.k=K\right)$. We also use the symbol $\boldsymbol{x}^{q}$ to denote the $n$-dimensional fuzzy region $\left[x^{1 . q_{1}} \ldots x^{n \cdot q_{n}}\right]^{T}$. For any given $x$, the value of membership function $m_{i . k}(x)$ determines the degree of $x$ in the fuzzy region $x^{i . k}$. In this paper, we only use triangular membership functions; one vertex lies at the center of the region and has membership value unity; the other two vertices lie at the centers of the two neighboring regions, respectively, and have membership values equal to zero.

Having defined the membership functions, we need to generate the fuzzy rules from given data pairs. We use if-then rules of the form IF $\boldsymbol{x}^{k}$ is in $\boldsymbol{x}^{q}$, THEN $\boldsymbol{y}=\boldsymbol{R}^{k}$, where $\boldsymbol{y}$ is the response of the rule. At the level of vector components it means

$$
\begin{aligned}
& \text { IF } x_{k .1} \text { is in } x^{1 . q_{1}} \text { AND } x_{k .2} \text { is in } x^{2 . q_{2}} \text { AND } \ldots \\
& \ldots \text { AND } x_{k . n} \text { is in } x^{n \cdot q_{n}} \\
& \text { THEN } \boldsymbol{y}=\boldsymbol{R}^{k}
\end{aligned}
$$

where $x_{k . i}, i=1, \ldots, n$ are components of vector $\boldsymbol{x}^{k}$. In general, it may happen that there are some conflicting rules, i.e., rules that have the same IF part, but a different THEN part. We resolve such conflicts by assigning a degree to each rule and accepting only the rule from a conflict group that has a maximum degree. A degree is assigned to a rule in the following way. For the rule "IF $x_{k .1}$ is in $x^{1 . q_{1}}$ AND $x_{k .2}$ is in $x^{2 . q_{2}}$ AND ... AND $x_{k . n}$ is in $x^{n . q_{n}}$ THEN $\boldsymbol{y}=\boldsymbol{R}^{k}$," the degree of this rule, denoted by $D\left(\boldsymbol{x}^{k}\right)$, is defined as

$$
D\left(\boldsymbol{x}^{k}, \boldsymbol{x}^{\boldsymbol{q}}\right)=\prod_{i=1}^{n} m_{i \cdot q_{i}}\left(x_{k . i}\right) .
$$

Having resolved the conflicts we have a set of nonconflicting rules, which we denote as $R_{i}, i=1,2, \ldots, L$. We denote by $\tilde{\boldsymbol{R}}_{s}: X_{R} \rightarrow R^{m}$ the output of our fuzzy system, which is determined using a centroid defuzzification

$$
\tilde{\boldsymbol{R}}_{s}(\boldsymbol{x})=\frac{\sum_{i=1}^{L} D\left(\boldsymbol{x}, \boldsymbol{x}^{i}\right) \boldsymbol{y}_{i}}{\sum_{i=1}^{L} D\left(\boldsymbol{x}, \boldsymbol{x}^{i}\right)}
$$

where $\boldsymbol{x}^{i}$ is an $n$-dimensional fuzzy region corresponding to the $i$ th rule, and $\boldsymbol{y}_{i}$ is the output of the $i$ th rule. 


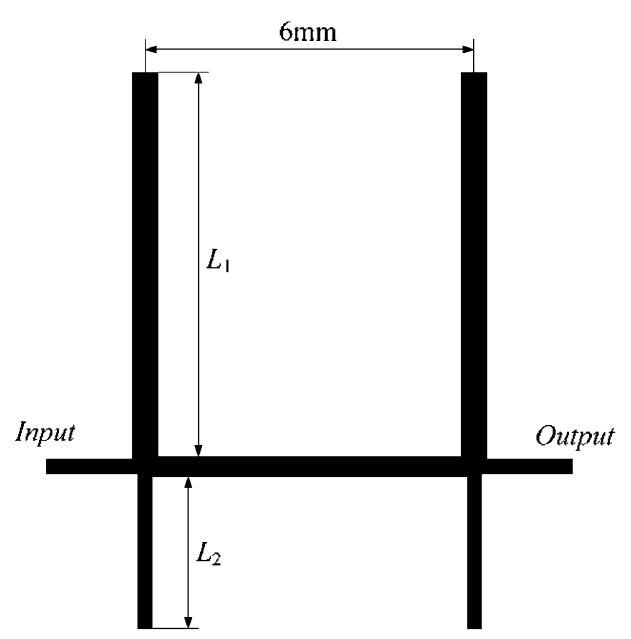

Fig. 2. Geometry of the second-order dual-behavior resonator filter [31].

Our surrogate model $\boldsymbol{R}_{s}$ combining the standard space-mapping surrogate $\overline{\boldsymbol{R}}_{s}$ and the fuzzy system $\tilde{\boldsymbol{R}}_{s}$ is defined as

$$
\boldsymbol{R}_{s}(\boldsymbol{x})=\overline{\boldsymbol{R}}_{s}(\boldsymbol{x})+\tilde{\boldsymbol{R}}_{s}(\boldsymbol{x}) .
$$

It should be noted that although the idea of combining space mapping with fuzzy systems is similar to the idea of combining space mapping with radial basis function interpolation described in [16], the latter technique is more difficult to implement. In particular, the problem of determining interpolation coefficients may be ill conditioned, especially if the number of base points is large. Moreover, the radial basis function interpolation may be very sensitive to control parameters, and typically, some sort of adjusting algorithm is necessary in order to find the proper values of these parameters. Fuzzy systems are free of these problems.

\section{EXAMPLES}

Here we compare the modeling accuracy for the standard space-mapping modeling methodology [11], space-mapping modeling with variable weight coefficients [14], space mapping with radial basis function interpolation [16], and the space mapping with fuzzy system interpolation described in Section II. In our comparison, we also include direct interpolation of the fine model data using a fuzzy system.

\section{A. Test Problem Description}

Problem 1: Second-order dual-behavior resonator microstrip filter [31] shown in Fig. 2. The fine model is simulated in FEKO [32]. The coarse model (see Fig. 3) is the circuit model implemented in Agilent ADS [33]. The design parameters are $\boldsymbol{x}=$ $\left[\begin{array}{ll}L_{1} & L_{2}\end{array}\right]^{T}$. The response vector consists of transmission coefficient $\left|S_{21}\right|$ in the frequency band $3 \leqslant \omega \leqslant 7 \mathrm{GHz}$ with samples taken every $200 \mathrm{MHz}$. The region of interest is defined by the reference point $\boldsymbol{x}^{0}=\left[\begin{array}{ll}8 & 4\end{array}\right]^{T} \mathrm{~mm}$ and the deviation $\delta=\left[\begin{array}{ll}1 & 0.5\end{array}\right]^{T}$ $\mathrm{mm}$.

Problem 2: Dual-band microstrip bandpass filter [34] shown in Fig. 4. The fine model is simulated in FEKO [32]. The coarse model (see Fig. 5) is the simplified equivalent-circuit model implemented in Agilent ADS [33]. The design parameters are

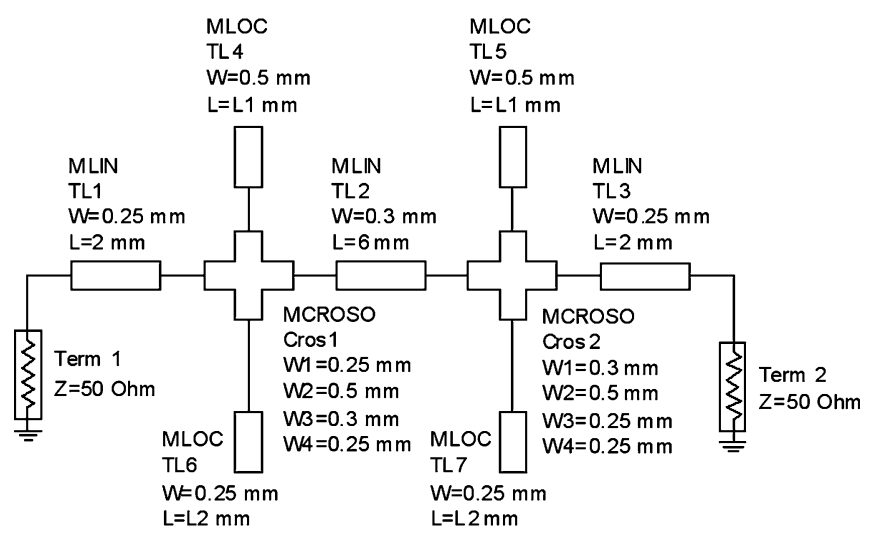

Fig. 3. Coarse model of the second-order dual-behavior resonator filter (Agilent ADS).

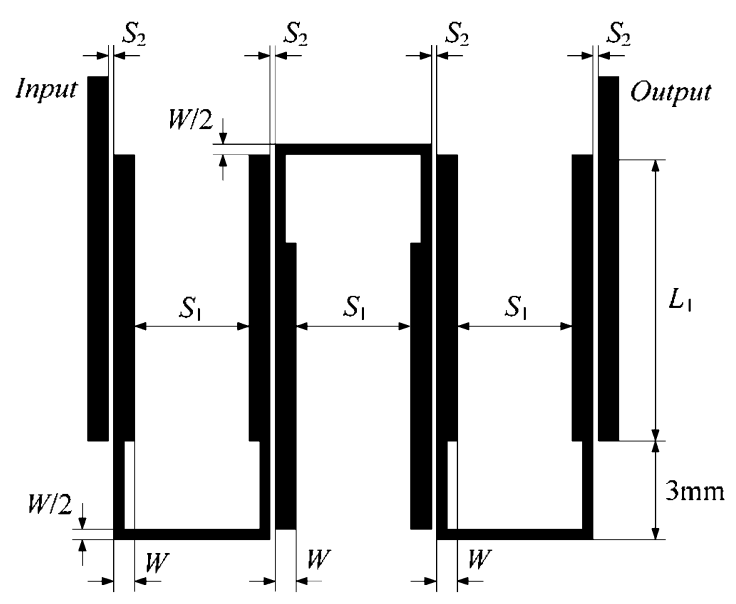

Fig. 4. Geometry of the dual-band microstrip bandpass filter [34].

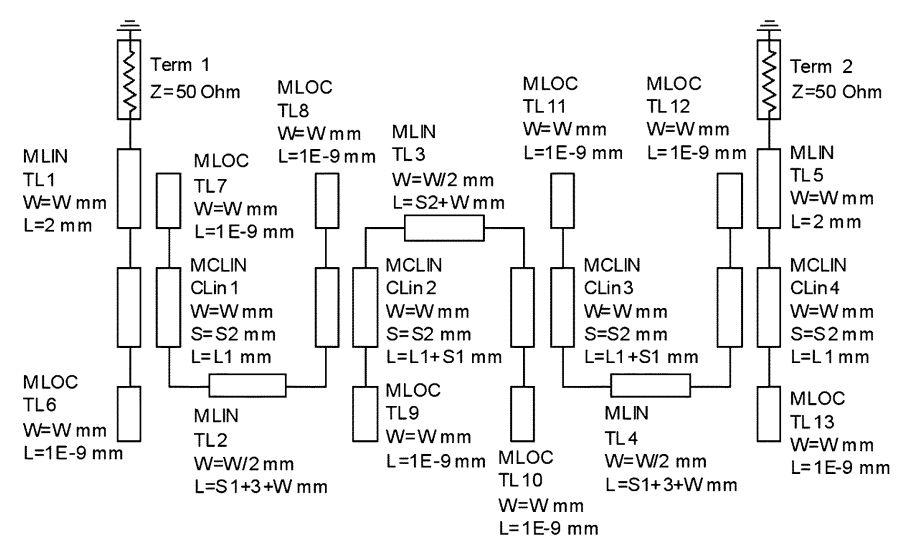

Fig. 5. Coarse model of the dual-band microstrip bandpass filter (Agilent ADS).

$\boldsymbol{x}=\left[\begin{array}{lll}L_{1} & S_{1} & S_{2}\end{array}\right]^{T}$. Parameter $W$ is set to $0.47 \mathrm{~mm}$. The response vector consists of transmission coefficient $\left|S_{21}\right|$ in the frequency range from 1.5 to $5.5 \mathrm{GHz}$. The reference point is

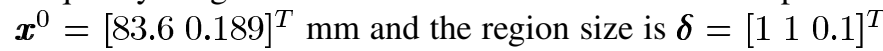
$\mathrm{mm}$.

Problem 3: Double-folded stub filter [1] shown in Fig. 6. The fine model is simulated with Sonnet's em [35] using a high-resolution grid with a $0.0254 \mathrm{~mm} \times 0.0254 \mathrm{~mm}$ cell size. 


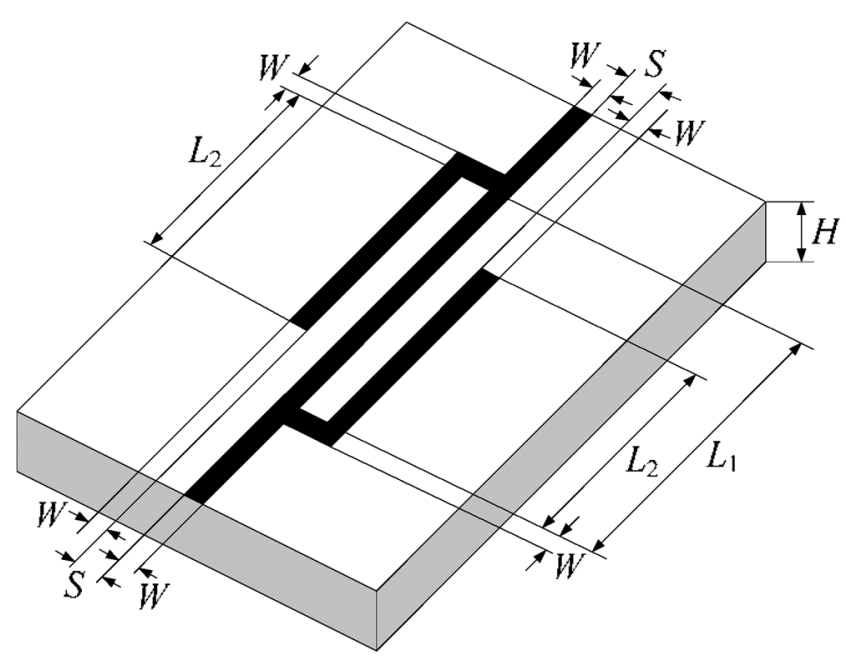

Fig. 6. Double-folded stub filter [1].

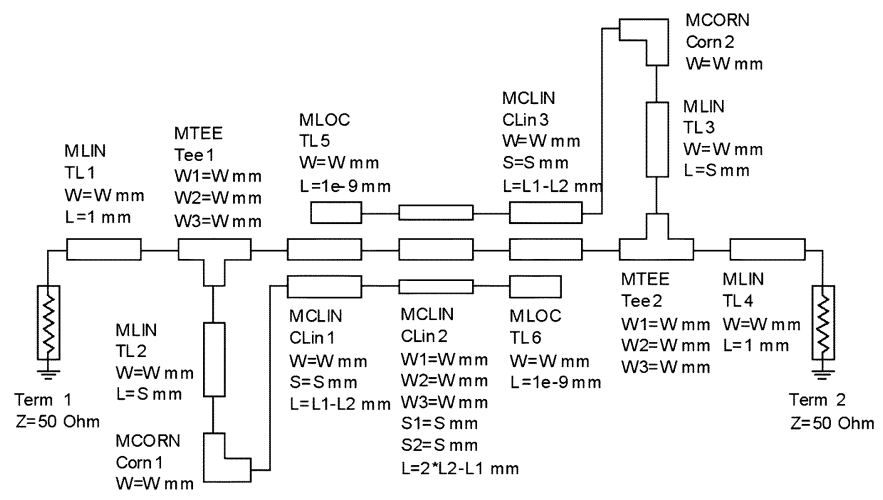

Fig. 7. Coarse model of the double-folded stub filter (Agilent ADS).

The coarse model (see Fig. 7) is the equivalent-circuit model implemented in Agilent ADS [33]. The design parameters are $\boldsymbol{x}=\left[\begin{array}{lll}L_{1} & L_{2} & S\end{array}\right]^{T}$. Parameter $W$ is set to $0.254 \mathrm{~mm}$. The response vector consists of transmission coefficient $\left|S_{21}\right|$ in the frequency range from 6 to $20 \mathrm{GHz}$. The reference point is $\boldsymbol{x}^{0}=\left[\begin{array}{lll}2.36 & 2.11 & 0.165\end{array}\right]^{T} \mathrm{~mm}$ and the region size is $\boldsymbol{\delta}=\left[\begin{array}{lll}0.152 & 0.076 & 0.076\end{array}\right]^{T} \mathrm{~mm}$.

\section{B. Experimental Setup}

For each of the test problems, we performed a number of numerical experiments using the standard space-mapping surrogate model, space mapping with variable-weight coefficients, space mapping with radial basis functions, space mapping with fuzzy systems, as well as direct fuzzy interpolation (i.e., the fuzzy system directly interpolating the fine model data). Table I shows details of the base sets used in our simulations. The base sets have growing numbers of points (and decreasing characteristic distance $\lambda$ ) in order to examine the dependence of the modeling error on the amount of fine model data used to create the model. Accuracy was tested using 30 test points randomly distributed in the region of interest. The error measure used was the $l_{2}$ norm of the difference between the fine model response and the corresponding surrogate model response.
TABLE I

Base Set Data For Test Problems 1-3

\begin{tabular}{|c|c|c|c|c|}
\hline $\begin{array}{c}\text { Test } \\
\text { Problem }\end{array}$ & $\begin{array}{c}\text { Base } \\
\text { Set }\end{array}$ & $\begin{array}{c}\text { Base Set } \\
\text { Description }\end{array}$ & $\begin{array}{l}\text { Number of } \\
\text { Base Points }\end{array}$ & $\lambda$ \\
\hline \multirow{3}{*}{1} & $X_{B 1}$ & Uniform mesh of density 3 & 9 & 0.50 \\
\hline & $X_{B 2}$ & Uniform mesh of density 4 & 16 & 0.38 \\
\hline & $X_{B 3}$ & Uniform mesh of density 8 & 64 & 0.19 \\
\hline \multirow{3}{*}{2} & $X_{B 1}$ & $\begin{array}{c}\text { Uniform mesh of density } 2 \\
\cup \text { star distribution }\end{array}$ & 15 & 0.57 \\
\hline & $X_{B 2}$ & Uniform mesh of density 3 & 27 & 0.47 \\
\hline & $X_{B 3}$ & Uniform mesh of density 4 & 64 & 0.35 \\
\hline \multirow{3}{*}{3} & $X_{B 1}$ & $\begin{array}{c}\text { Uniform mesh of density } 2 \\
\cup \text { star distribution }\end{array}$ & 15 & 3.24 \\
\hline & $X_{B 2}$ & Uniform mesh of density 3 & 27 & 2.67 \\
\hline & $X_{B 3}$ & Uniform mesh of density 4 & 64 & 2.00 \\
\hline
\end{tabular}

TABLE II

Modeling Results for TeSt PROBlem 1. Verification FOR 30 RANDOM TEST POINTS

\begin{tabular}{|c|c|c|c|c|}
\hline Model & $\begin{array}{c}\text { Base } \\
\text { set }\end{array}$ & $\begin{array}{c}\text { Average } \\
\text { Error }\end{array}$ & $\begin{array}{l}\text { Maximum } \\
\text { Error }\end{array}$ & $\begin{array}{c}\text { Standard } \\
\text { Deviation }\end{array}$ \\
\hline Standard space mapping & & 0.0238 & 0.0274 & 0.0020 \\
\hline Space mapping $\mathrm{m}$ & & 0.0129 & 0.0289 & \\
\hline Space mapping model [16] & $X_{B 1}$ & 0.0131 & 0.0218 & 0.0049 \\
\hline Space mapping model (2)-(8) & & 0.0136 & 0.0227 & 0.0048 \\
\hline Direct fuzzy & & 0.18 & & \\
\hline Standard sp & & 0.0225 & 264 & \\
\hline Space mapping model [14] & & 0.0112 & & 68 \\
\hline Space mapping model [16] & $X_{B 2}$ & 0.0094 & 177 & 0.0042 \\
\hline Space mapping model (2)-(8) & & 0.0100 & 0.0174 & 0.0038 \\
\hline Direct fuzzy interpolation & & 0.1163 & 0.1957 & 0.0430 \\
\hline & & & & \\
\hline Space mapping 1 & & 0.0062 & 174 & 045 \\
\hline Space mapping model [16] & $X_{B 3}$ & 0.0024 & 0.0051 & 0.0010 \\
\hline Space mapping model (2)-(8) & & 0.0026 & 0.0054 & 0.0011 \\
\hline Direct fuzzy interpolation & & 0.0244 & 0.0492 & 0.0118 \\
\hline
\end{tabular}

TABLE III

Modeling Results for Test PROBlem 2. Verification FOR 30 RANDOM TEST POINTS

\begin{tabular}{|c|c|c|c|c|}
\hline Model & $\begin{array}{c}\text { Base } \\
\text { set }\end{array}$ & $\begin{array}{l}\text { Average } \\
\text { Error }\end{array}$ & $\begin{array}{l}\text { Maximum } \\
\text { Error }\end{array}$ & $\begin{array}{l}\text { Standard } \\
\text { Deviation }\end{array}$ \\
\hline Standard space mapping & & 14.86 & 22.22 & 2.70 \\
\hline Space mapping model [14] & & 11.79 & 20.10 & 2.73 \\
\hline Space mapping model [16] & $X_{B 1}$ & 12.00 & 16.81 & 2.21 \\
\hline Space mapping model (2)-(8) & & 11.90 & 22.84 & 2.92 \\
\hline Direct fuzzy interpolation & & 21.12 & 34.37 & 6.07 \\
\hline Standard space mapping & & 14.49 & 21.85 & 2.90 \\
\hline Space mapping model [14] & & 9.91 & 18.52 & 3.40 \\
\hline Space mapping model [16] & $X_{B 2}$ & 10.83 & 14.60 & 2.29 \\
\hline Space mapping model (2)-(8) & & 9.83 & 14.08 & 2.21 \\
\hline Direct fuzzy interpolation & & 14.42 & 23.25 & 3.90 \\
\hline Standard space mapping & & 13.65 & 21.02 & 3.05 \\
\hline Space mapping model [14] & & 7.72 & 16.04 & 2.83 \\
\hline Space mapping model [16] & $X_{B 3}$ & 9.67 & 18.42 & 3.84 \\
\hline Space mapping model (2)-(8) & & 6.01 & 11.50 & 2.33 \\
\hline Direct fuzzy interpolation & & 8.19 & 15.75 & 3.31 \\
\hline
\end{tabular}

\section{Numerical Results and Discussion}

Tables II-IV show numerical results (error statistics) for the considered models with all the base sets considered. Figs. 8-13 show error plots, i.e., the modulus of the difference between the fine model and the corresponding surrogate model response 
TABLE IV

MODELING Results FOR TEST PROBLEM 3. VerificATION FOR 30 RANDOM TEST POINTS

\begin{tabular}{ccccc}
\hline \hline \multirow{2}{*}{ Model } & Base & Average & Maximum & Standard \\
& set & Error & Error & Deviation \\
\hline Standard space mapping & & 0.0692 & 0.1087 & 0.0177 \\
Space mapping model [14] & & 0.0503 & 0.0789 & 0.0140 \\
Space mapping model [16] & $X_{B 1}$ & 0.0306 & 0.0630 & 0.0131 \\
Space mapping model (2)-(8) & & 0.0314 & 0.0647 & 0.0144 \\
Direct fuzzy interpolation & 0.1447 & 0.5090 & 0.1061 \\
\hline Standard space mapping & 0.0691 & 0.1084 & 0.0176 \\
Space mapping model [14] & 0.0448 & 0.0692 & 0.0132 \\
Space mapping model [16] & $X_{B 2}$ & 0.0284 & 0.0511 & 0.0104 \\
Space mapping model (2)-(8) & 0.0242 & 0.0418 & 0.0088 \\
Direct fuzzy interpolation & 0.0612 & 0.0939 & 0.0205 \\
\hline Standard space mapping & 0.0676 & 0.1056 & 0.0176 \\
Space mapping model [14] & 0.0357 & 0.0587 & 0.0118 \\
Space mapping model [16] & $X_{B 3}$ & 0.0154 & 0.0272 & 0.0062 \\
Space mapping model (2)-(8) & 0.0143 & 0.0267 & 0.0062 \\
Direct fuzzy interpolation & 0.0262 & 0.0500 & 0.0118 \\
\hline \hline
\end{tabular}

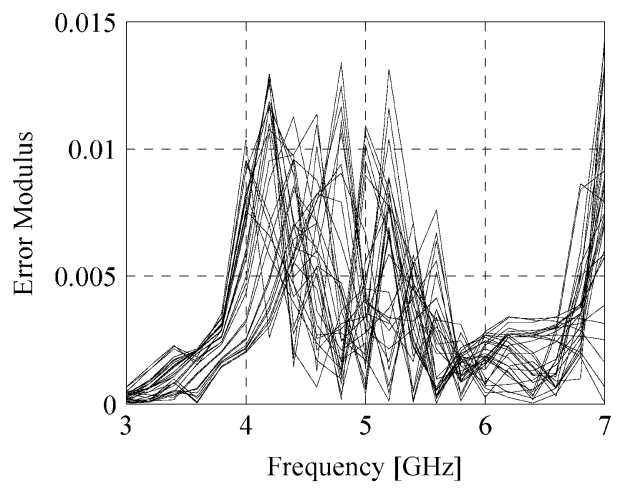

Fig. 8. Test problem 1: error plots for the standard space-mapping model.

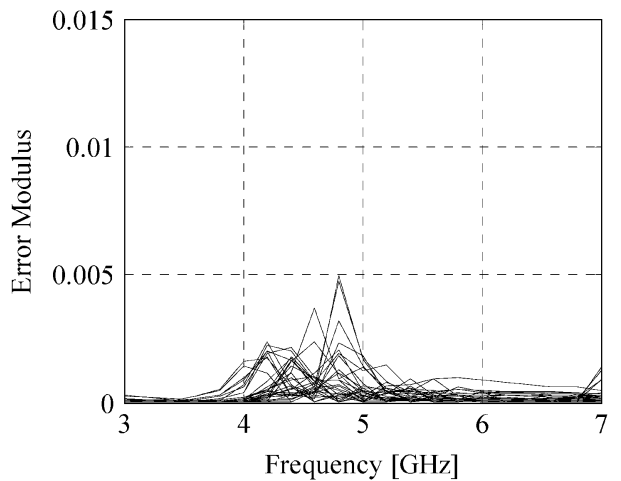

Fig. 9. Test problem 1: error plots for space mapping combined with the fuzzy system.

versus frequency, for the standard space-mapping model and the space mapping with fuzzy interpolation; data obtained for the base set $X_{B 3}$ was used in all cases. Figs. 14-16 show the average modeling error versus the characteristic distance $\lambda$.

It follows from the results that the modeling accuracy provided by the new model (2)-(8) is comparable with or better than the accuracy of space mapping enhanced by radial basis function interpolation. All the other space-mapping approaches, as well as the direct fuzzy interpolation, are clearly outperformed by these two techniques. As expected, the accuracy of the standard space-mapping model is almost independent of the density

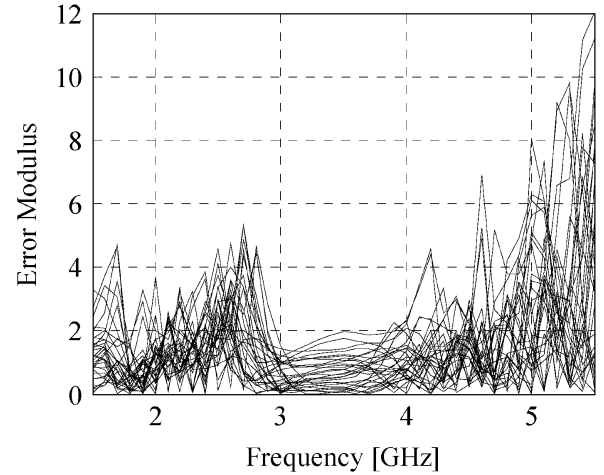

Fig. 10. Test problem 2: error plots for the standard space-mapping model.

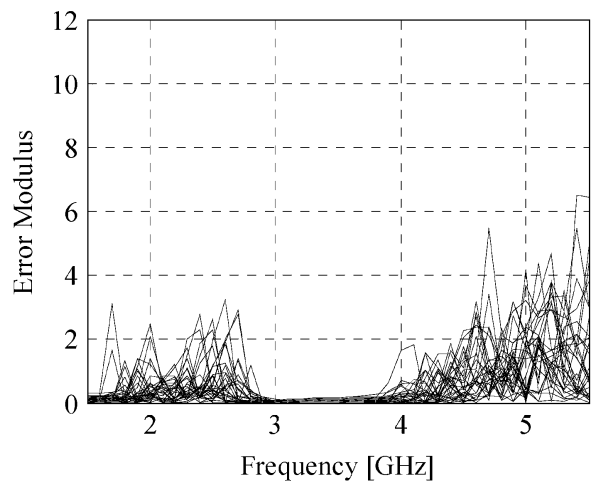

Fig. 11. Test problem 2: error plots for space mapping combined with the fuzzy system.

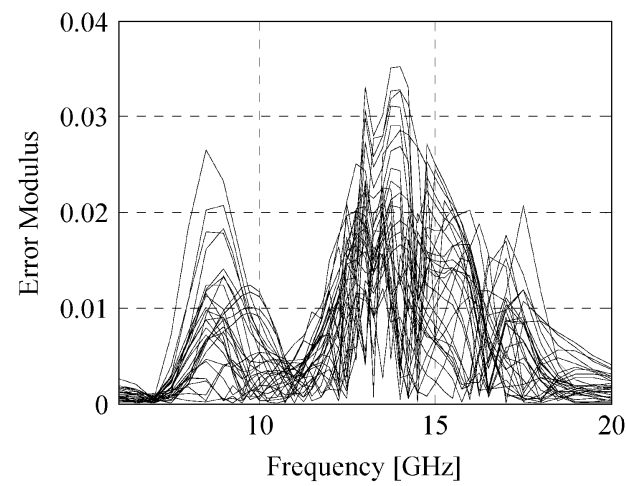

Fig. 12. Test problem 3: error plots for the standard space-mapping model.

of the base set. Other approaches exhibit improvement of the modeling quality with decrease of the characteristic distance of the base set.

It should be mentioned that the computational cost of the model (2)-(8) is almost the same as the cost of the coarse model because once the parameters are established (including fuzzy rules), evaluation of formula (7) (defuzzification) is very fast. Space mapping combined with radial basis function interpolation exhibits similar advantages with respect to computational efficiency, although, as mentioned in Section I, it has some inherent problems such as sensitivity to the control parameters and the possibility of the parameter calculation being ill conditioned. On the other hand, space mapping with variable weight coefficients, which performs well in terms of accuracy, suffers 


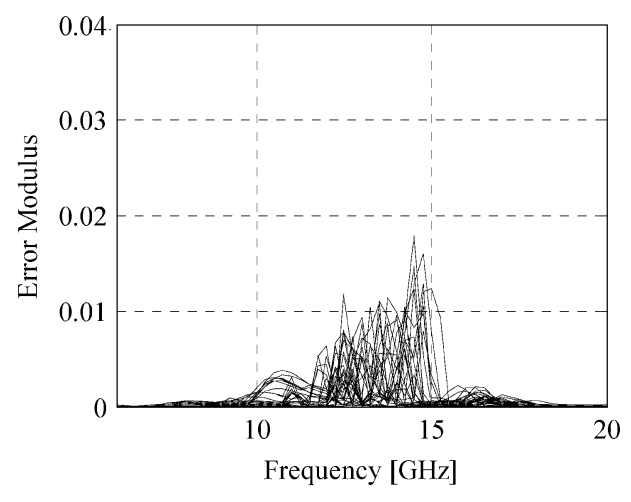

Fig. 13. Test problem 3: error plots for space mapping combined with the fuzzy system.

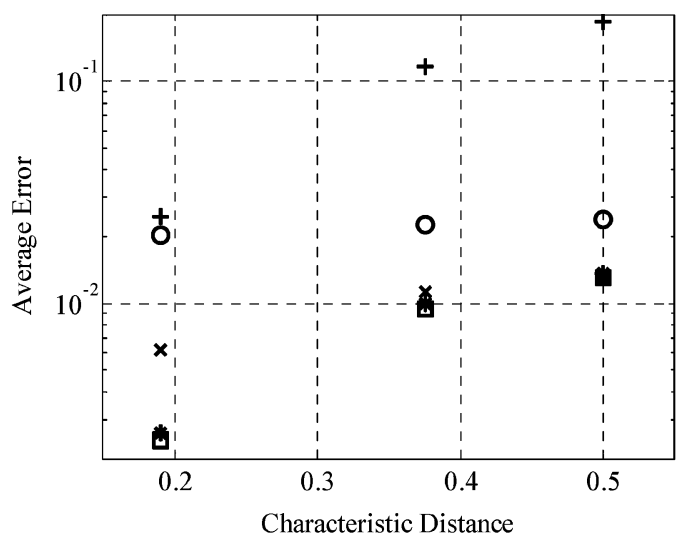

Fig. 14. Test problem 1: average modeling error versus characteristic distance $\lambda$. Data for the standard space-mapping model (o), space mapping with variable weight coefficients $(x)$, space mapping with radial basis functions $(\square)$, space mapping with the fuzzy system $(*)$, and direct fuzzy interpolation $(+)$.

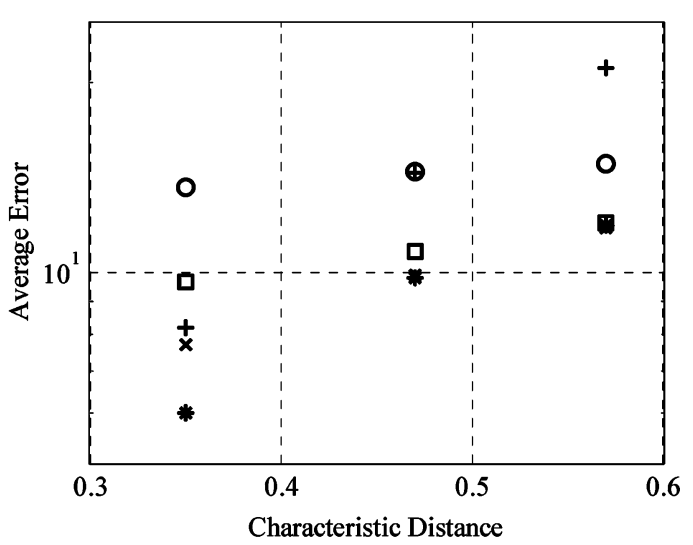

Fig. 15. Test problem 2: average modeling error versus characteristic distance $\lambda$. Data for the standard space-mapping model (o), space mapping with variable weight coefficients $(x)$, space mapping with radial basis functions $(\square)$, space mapping with the fuzzy system $(*)$, and direct fuzzy interpolation $(+)$.

from computational overhead related to separate parameter extractions required for each evaluation of the model.

Overall, the presented combination of space mapping and fuzzy systems seems to be a robust alternative to the existing modeling techniques based on space mapping.

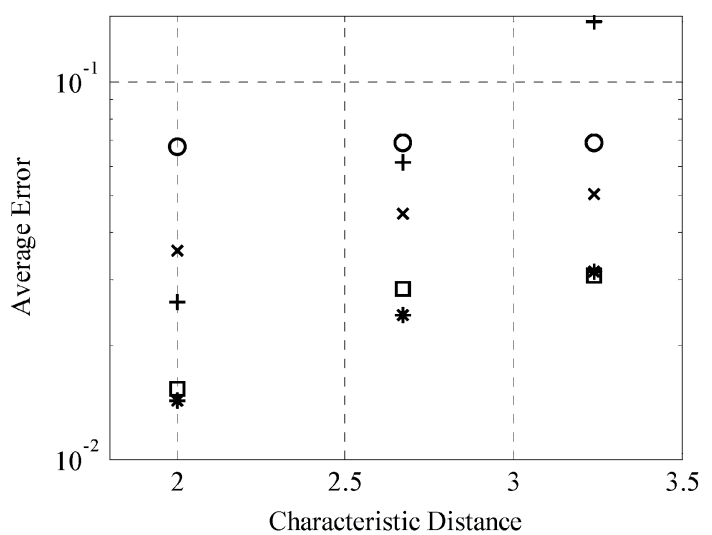

Fig. 16. Test problem 3: average modeling error versus characteristic distance $\lambda$. Data for the standard space-mapping model (o), space mapping with variable weight coefficients $(x)$, space mapping with radial basis functions $(\square)$, space mapping with the fuzzy system $(*)$, and direct fuzzy interpolation $(+)$.

\section{Fuzzy Systems With Alternative Membership Functions}

The fuzzy system described in Section II uses triangle membership functions. This kind of model has an interpolation property provided that the base set is a uniform mesh and that base points are located at the centers of the membership functions. Here, we compare the accuracy of the space-mapping surrogate model (2)-(8) with the space-mapping model using the fuzzy system with Gaussian and Z-shaped membership functions. These functions can be beneficial in some cases, especially if the fine model response exhibits highly nonlinear behavior. Also, the resulting surrogate model is smooth, which is not the case for triangular functions. The Gaussian membership function is given by $m_{i . j}(x)=\exp \left(-\left(x-x^{i . j}\right)^{2} / 2 \sigma^{2}\right)$, with $\sigma$ being the control parameter, which must be optimized in general in order to obtain the best performance. The Z-shaped function is defined, for $x^{i . j} \leqslant x \leqslant x^{i . j+1}$, as $m_{i . j}(x)=1-2((x-$ $\left.\left.x^{i . j}\right) / s\right)^{2}$ if $x-x^{i . j} \leqslant s$, and $m_{i . j}(x)=2\left(1-\left(x-x^{i . j}\right) / s\right)^{2}$ if $x-x^{i . j}>s$, where $s=x^{i . j+1}-x^{i . j}$. Definitions for other intervals are similar.

Table $\mathrm{V}$ presents a comparison of the average modeling error for Problems $1-3$ with the base sets $X_{B 1}, X_{B 2}$, and $X_{B 3}$. It is seen that the performance of the surrogate model is very similar for all membership functions considered. It should be noted that the model using the fuzzy system with unoptimized Gaussian membership functions exhibits the worst performance, which is most likely because this model does not exhibit an interpolation property.

\section{Possible Extensions of FuZzy SPACE MapPing}

Apart from the fuzzy space-mapping surrogate model (8) described in Section II, it is possible to employ fuzzy systems to approximate the space-mapping parameters in a regular space-mapping model $\overline{\boldsymbol{R}}_{s}$. In order to discuss this concept, we will use the following notation. Let $\overline{\boldsymbol{R}}_{s}=\overline{\boldsymbol{R}}_{s}(\boldsymbol{x}, \boldsymbol{p})$ be a compact way of denoting the space-mapping surrogate model, where $\boldsymbol{p}$ is a vector of the model parameters. Let $\boldsymbol{F}\left(\cdot, \boldsymbol{y}, X_{B}\right)=\boldsymbol{F}\left(\cdot,\left\{\left(\boldsymbol{x}^{k}, \boldsymbol{y}^{k}\right): \boldsymbol{x}^{k} \in X_{B}\right\}\right)$ denote a fuzzy system that approximates variable $y$ and is built using data 
TABLE V

MODELING ACCURACY FOR SPACE-MAPPING SURROGATE With FUZZY SYSTEM USING DIFFERENT MEMBERSHIP FUNCTIONS

\begin{tabular}{ccccc}
\hline \hline \multirow{2}{*}{ Membership Function } & Base & \multicolumn{3}{c}{ Average Modeling Error } \\
\cline { 3 - 5 } & Set & Problem 1 & Problem 2 & Problem 3 \\
\hline Triangle & & 0.0136 & 11.90 & 0.0314 \\
Gaussian $^{\#}$ & & 0.0182 & 12.99 & 0.0424 \\
Optimized Gaussian $^{*}$ & $X_{B 1}$ & 0.0136 & 11.73 & 0.0305 \\
Z-shaped $^{\text {Triangle }}$ & & 0.0137 & 12.77 & 0.0350 \\
\hline Gaussian & & 0.0100 & 9.83 & 0.0242 \\
Optimized Gaussian $^{*}$ & $X_{B 2}$ & 0.0150 & 11.95 & 0.0417 \\
Z-shaped & & 0.0099 & 9.76 & 0.0246 \\
Triangle & & 0.0099 & 10.39 & 0.0261 \\
\hline Gaussian & & 0.0026 & 6.01 & 0.0143 \\
Optimized Gaussian $^{*}$ & $X_{B 3}$ & 0.0066 & 9.60 & 0.0309 \\
Z-shaped & & 0.0028 & 6.05 & 0.0150 \\
\hline
\end{tabular}

${ }^{\#}$ Gaussian membership function with $\sigma=1$.

*Gaussian membership function with optimized value of $\sigma$.

pairs $\left(\boldsymbol{x}^{k}, \boldsymbol{y}^{k}\right)$ with $\boldsymbol{x}^{k}$ from a given base set $X_{B} ; \boldsymbol{F}\left(\boldsymbol{x}, \boldsymbol{y}, X_{B}\right)$ denotes the response of the fuzzy system at point $\boldsymbol{x}$.

Using this notation, fuzzy space-mapping model (8) can be written as $\boldsymbol{R}_{s}(\boldsymbol{x})=\overline{\boldsymbol{R}}_{s}(\boldsymbol{x}, \boldsymbol{p})+\boldsymbol{F}\left(\boldsymbol{x}, \boldsymbol{R}, X_{B}\right)$. Thus, we can call it the fuzzy output space-mapping model. As mentioned before, one of the possible extensions of the model (8) is to use a fuzzy system not only to approximate the differences between the fine model and the regular space-mapping model, but also to approximate space-mapping parameters in the regular spacemapping model. Using our notation, the extended model has the following form:

$$
\widehat{\boldsymbol{R}}_{s}(\boldsymbol{x})=\overline{\boldsymbol{R}}_{\boldsymbol{s}}\left(\boldsymbol{x}, \boldsymbol{F}\left(\boldsymbol{x}, \boldsymbol{p}, X_{B 1}\right)\right)+\boldsymbol{F}\left(\boldsymbol{x}, \boldsymbol{R}, X_{B 2}\right)
$$

where, in general, both base sets $X_{B 1}$ and $X_{B 2}$ can be different, although in practice they should be the same in order to efficiently use the available fine model data. Another possibility is to use a fuzzy system only to approximate the space-mapping parameters, which would give the following model:

$$
\breve{R}_{s}(x)=\overline{\boldsymbol{R}}_{s}\left(\boldsymbol{x}, \boldsymbol{F}\left(\boldsymbol{x}, \boldsymbol{p}, X_{B 1}\right)\right)
$$

Using space-mapping terminology, model (10) is the fuzzy input space-mapping surrogate, while model (9) is the fuzzy input and output space-mapping surrogate. It should be noted that model (10) does not fully use available fine model information, and, therefore, model (9) is expected to outperform model (10) when using the same base set $X_{B 1}$.

An extended fuzzy space-mapping model (9) may seem attractive, however, there is an issue that makes its actual usefulness questionable. In order to model the space-mapping parameters with a fuzzy system, one has to extract the optimal set of parameters for each of base points from $X_{B 1}$ and then hope to retrieve the optimal set of parameters for any other point from the region of interest using a fuzzy system. The problem is that, typically, the optimal space-mapping parameter set corresponding to a given design variable vector is nonlinearly dependent on the vector and it might be very difficult to model this dependency with a fuzzy system (or, more generally, with any other approach).

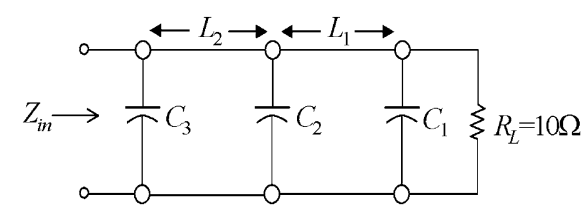

(a)

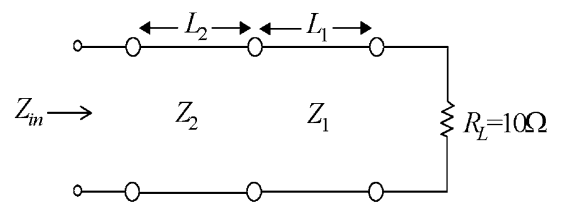

(b)

Fig. 17. (a) Fine and (b) coarse model, two-section capacitively loaded impedance transformer [36]

TABLE VI

BASE SET DATA FOR TWO-SECTION TRANSFORMER EXAMPLE

\begin{tabular}{cccc}
\hline \hline Base set & Description & Number of points & $\lambda$ \\
\hline$X_{B 1}$ & Uniform mesh of density 3 & 9 & 5.12 \\
$X_{B 2}$ & Uniform mesh of density 5 & 25 & 3.07 \\
$X_{B 3}$ & Uniform mesh of density 7 & 49 & 2.19 \\
$X_{B 4}$ & Uniform mesh of & 100 & 1.53 \\
& density 10 & \\
\hline \hline
\end{tabular}

TABLE VII

Modeling Results For Two-SECTION TRANSFORMER EXAMPLE. AVERAGE ERROR FOR 30 RANDOM TEST POINTS

\begin{tabular}{ccccc}
\hline \hline \multirow{2}{*}{ Model } & \multicolumn{4}{c}{ Base set } \\
& $X_{\boldsymbol{B} 1}$ & $X_{\boldsymbol{B} 2}$ & $X_{\boldsymbol{B} 3}$ & $X_{\boldsymbol{B} 4}$ \\
\hline Fuzzy space mapping (8) & 0.0129 & 0.0039 & 0.0024 & 0.0012 \\
Fuzzy space mapping (9) & 0.0204 & 0.0115 & 0.0081 & 0.0046 \\
Fuzzy space mapping (10) & 0.0209 & 0.0137 & 0.0095 & 0.0049 \\
\hline \hline
\end{tabular}

For illustrative purposes, consider a capacitively loaded $10: 1$ two-section impedance transformer example [36]. The "coarse" model and "fine" model, both implemented in MATLAB, are an ideal two-section transmission line and a capacitively loaded transmission line, as shown in Fig. 17. The electrical lengths $L_{1}$ and $L_{2}$ are chosen as design parameters. The response vector consists of reflection coefficient $\left|S_{11}\right|$ in the frequency range from 0.5 to $1.5 \mathrm{GHz}$. The reference point is $x^{0}=\left[74.25^{\circ} 79.24^{\circ}\right]^{T}$. We consider the region of interest defined by a $10 \%$ deviation from $\boldsymbol{x}^{0}$.

The base sets considered for this problem are shown in Table VI. In Table VII, we compare modeling accuracy for the space-mapping model (8), as well as fuzzy space-mapping models (9) and (10). Space-mapping parameters for models (9) and (10) were obtained using space mapping with the variable weight coefficient modeling technique [14].

It follows from the results that although models (9) and (10) retain the pattern of improving accuracy with growing density of the base set, their performance is not as good as for model (8).

\section{CONCLUSION}

A modeling methodology has been presented that combines the standard space-mapping technique and fuzzy interpolation. The new methodology can efficiently handle any base set. As 
with most of the recent space-mapping-based surrogate modeling techniques, it has the property of increasing modeling accuracy when the number of base points increases. Examples have demonstrated the robustness of our method. It follows that the new approach provides modeling accuracy comparable with or better than the recently published space mapping enhanced by radial basis function interpolation, and outperforms any other space-mapping approach. The new technique is easy to implement and computationally efficient.

\section{ACKNOWLEDGMENT}

The authors thank Sonnet Software Inc., Syracuse, NY, for $\boldsymbol{e m}$ and Agilent Technologies, Santa Rosa, CA, for making ADS available.

\section{REFERENCES}

[1] J. W. Bandler, R. M. Biernacki, S. H. Chen, P. A. Grobelny, and R. H. Hemmers, "Space mapping technique for electromagnetic optimization," IEEE Trans. Microw. Theory Tech., vol. 42, no. 12, pp. 536-544, Dec. 1994.

[2] J. W. Bandler, Q. S. Cheng, S. A. Dakroury, A. S. Mohamed, M. H. Bakr, K. Madsen, and J. Søndergaard, "Space mapping: The state of the art," IEEE Trans. Microw. Theory Tech., vol. 52, no. 1, pp. 337-361, Jan. 2004.

[3] S. Koziel, J. W. Bandler, and K. Madsen, "A space mapping framework for engineering optimization: Theory and implementation," IEEE Trans. Microw. Theory Tech., vol. 54, no. 10, pp. 3721-3730, Oct. 2006.

[4] D. Echeverria and P. W. Hemker, "Space mapping and defect correction," Int. Math. J. Comput. Methods Appl. Math., vol. 5, no. 2, pp. 107-136, 2005.

[5] H.-S. Choi, D. H. Kim, I. H. Park, and S. Y. Hahn, "A new design technique of magnetic systems using space mapping algorithm," IEEE Trans. Magn., vol. 37, no. 5, pp. 3627-3630, Sep. 2001.

[6] M. A. Ismail, D. Smith, A. Panariello, Y. Wang, and M. Yu, "EMbased design of large-scale dielectric-resonator filters and multiplexers by space mapping," IEEE Trans. Microw. Theory Tech., vol. 52, no. 1, pp. 386-392, Jan. 2004.

[7] S. Amari, C. LeDrew, and W. Menzel, "Space-mapping optimization of planar coupled-resonator microwave filters," IEEE Trans. Microw. Theory Tech., vol. 54, no. 5, pp. 2153-2159, May 2006.

[8] J. E. Rayas-Sánchez and V. Gutiérrez-Ayala, "EM-based Monte Carlo analysis and yield prediction of microwave circuits using linear-input neural-output space mapping," IEEE Trans. Microw. Theory Tech., vol. 54, no. 12, pp. 4528-4537, Dec. 2006.

[9] M. H. Bakr, J. W. Bandler, and N. Georgieva, "Modeling of microwave circuits exploiting space derivative mapping," in IEEE MTT-S Int. Microw. Symp. Dig., Anaheim, CA, Jun. 1999, pp. 715-718.

[10] J. W. Bandler, N. Georgieva, M. A. Ismail, J. E. Rayas-Sánchez, and Q. J. Zhang, "A generalized space mapping tableau approach to device modeling," IEEE Trans. Microw. Theory Tech., vol. 49, no. 1, pp. 67-79, Jan. 2001

[11] S. Koziel, J. W. Bandler, A. S. Mohamed, and K. Madsen, "Enhanced surrogate models for statistical design exploiting space mapping technology," in IEEE MTT-S Int. Microw. Symp. Dig., Long Beach, CA, Jun. 2005, pp. 1609-1612.

[12] J. W. Bandler, Q. S. Cheng, and S. Koziel, "Simplified space mapping approach to enhancement of microwave device models," Int. J. RF Microw. Comput.-Aided Eng., vol. 16, no. 5, pp. $518-535,2006$.

[13] J. C. Rautio, "A space mapped model of thick, tightly coupled conductors for planar electromagnetic analysis," IEEE Micro, vol. 5, no. 3, pp. 62-72, Sep. 2004.

[14] S. Koziel and J. W. Bandler, "Space-mapping-based modeling utilizing parameter extraction with variable weight coefficients and a data base," in IEEE MTT-S Int. Microw. Symp. Dig., San Francisco, CA, Jun. 2006, pp. $1763-1766$.
[15] S. Koziel, J. W. Bandler, and K. Madsen, "Theoretical justification of space-mapping-based modeling utilizing a data base and on-demand parameter extraction," IEEE Trans. Microw. Theory Tech., vol. 54, no. 12, pp. 4316-4322, Dec. 2006.

[16] S. Koziel and J. W. Bandler, "Microwave device modeling using space-mapping and radial basis functions," in IEEE MTT-S Int. Microw. Symp. Dig., Honolulu, HI, 2007, pp. 799-802.

[17] L. Zhang, J. J. Xu, M. Yagoub, R. T. Ding, and Q. J. Zhang, "Neuro-space mapping technique for nonlinear device modeling and large signal simulation," in IEEE MTT-S Int. Microw. Symp. Dig., Philadelphia, PA, Jun. 2003, pp. 173-176.

[18] V. K. Devabhaktuni, B. Chattaraj, M. C. E. Yagoub, and Q.-J. Zhang, "Advanced microwave modeling framework exploiting automatic model generation, knowledge neural networks, and space mapping," IEEE Trans. Microw. Theory Tech., vol. 51, no. 7, pp. 1822-1833, Jul. 2003.

[19] L. Zhang, J. Xu, M. C. E. Yagoub, R. Ding, and Q.-J. Zhang, "Efficient analytical formulation and sensitivity analysis of neuro-space mapping for nonlinear microwave device modeling," IEEE Trans. Microw. Theory Tech., vol. 53, no. 9, pp. 2752-2767, Sep. 2005.

[20] P. Burrascano, M. Dionigi, C. Fancelli, and M. Mongiardo, "A neural network model for CAD and optimization of microwave filters," in IEEE MTT-S Int. Microw. Symp. Dig., Baltimore, MD, 1998, pp. $13-16$.

[21] S. F. Peik, R. R. Mansour, and Y. L. Chow, "Multidimensional Cauchy method and adaptive sampling for an accurate microwave circuit modeling," IEEE Trans. Microw. Theory Tech., vol. 46, no. 12, pp. 2364-2371, Dec. 1998.

[22] X. Ding, V. K. Devabhaktuni, B. Chattaraj, M. C. E. Yagoub, M. Doe, J. J. Xu, and Q. J. Zhang, "Neural network approaches to electromagnetic based modeling of passive components and their applications to high-frequency and high-speed nonlinear circuit optimization," IEEE Trans. Microw. Theory Tech., vol. 52, no. 1, pp. 436-449, Jan. 2004.

[23] T. W. Simpson, J. Peplinski, P. N. Koch, and J. K. Allen, "Metamodels for computer-based engineering design: Survey and recommendations," Eng. Comput., vol. 17, no. 2, pp. 129-150, Jul. 2001.

[24] N. V. Queipo, R. T. Haftka, W. Shyy, T. Goel, R. Vaidynathan, and P. K. Tucker, "Surrogate-based analysis and optimization," Progress Aerosp. Sci., vol. 41, no. 1, pp. 1-28, Jan. 2005.

[25] A. A. Mullur and A. Messac, "Metamodeling using extended radial basis functions: A comparative approach," Eng. Comput., vol. 21, no. 3, pp. 203-217, Apr. 2006.

[26] T. W. Simpson, T. M. Maurey, J. J. Korte, and F. Mistree, "Kriging models for global approximation in simulation-based multidisciplinary design optimization," AIAA J., vol. 39, no. 12, pp. 2233-2241, Dec. 2001.

[27] V. Miraftab and R. R. Mansour, "Computer-aided tuning of microwave filters using fuzzy logic," IEEE Trans. Microw. Theory Tech., vol. 50, no. 12, pp. 2781-2788, Dec. 2002.

[28] V. Miraftab and R. R. Mansour, "A robust fuzzy-logic technique for computer-aided diagnosis of microwave filters," IEEE Trans. Microw. Theory Tech., vol. 52, no. 1, pp. 450-456, Jan. 2004.

[29] V. Miraftab and R. R. Mansour, "EM-based microwave circuit design using fuzzy logic techniques," Proc. Inst. Elect. Eng.-Microw., Antennas, Propag., vol. 153, no. 6, pp. 495-501, Dec. 2006.

[30] L.-X. Wang and J. M. Mendel, "Generating fuzzy rules by learning from examples," IEEE Trans. Syst., Man, Cybern., vol. 22, no. 6, pp. 1414-1427, Nov./Dec. 1992.

[31] A. Manchec, C. Quendo, J. F. Favennec, E. Rius, and C. Person, "Synthesis of capacitive-coupled dual-behavior resonator (CCDBR) filters," IEEE Trans. Microw. Theory Tech., vol. 54, no. 6, pp. 2346-2355, Jun. 2006.

[32] “FEKO ${ }^{\circledR}$ User's Manual, Suite 4.2,” EM Softw. Syst. S.A. (Pty) Ltd., Stellenbosch, South Africa, Jun. 2004. [Online]. Available: http://www.feko.info

[33] Agilent ADS. ver. 2003C, Agilent Technol., Santa Rosa, CA, 2003.

[34] Y. P. Zhang and M. Sun, "Dual-band microstrip bandpass filter using stepped-impedance resonators with new coupling schemes," IEEE Trans. Microw. Theory Tech., vol. 54, no. 10, pp. 3779-3785, Oct. 2006

[35] em. ver. 11.52, Sonnet Software Inc., North Syracuse, NY, 2007.

[36] M. H. Bakr, J. W. Bandler, K. Madsen, and J. Søndergaard, "An introduction to the space mapping technique," Optim. Eng., vol. 2, no. 4, pp. 369-384, Dec. 2001. 


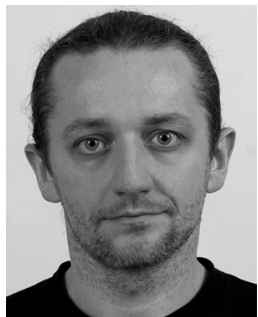

Slawomir Koziel (M'03-SM'07) received the M.Sc. and Ph.D. degrees in electronic engineering, M.Sc. degrees in theoretical physics and in mathematics, and $\mathrm{Ph} . \mathrm{D}$. degree in mathematics from the Gdansk University of Technology, Gdansk, Poland, in 1995, 2000, 2000 and 2002, and 2003, respectively.

$\mathrm{He}$ is currently a Research Associate with the Department of Electrical and Computer Engineering, McMaster University, Hamilton, ON, Canada. He has authored or coauthored over 100 papers. His research interests include space mapping, circuit theory, analog signal processing, evolutionary computation, and numerical analysis.

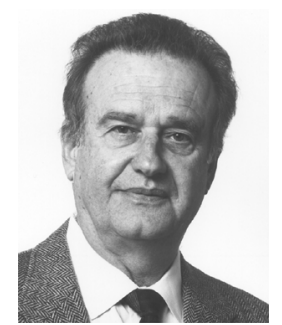

John W. Bandler (S'66-M'66-SM'74-F'78LF'06) studied at Imperial College. He received the B.Sc. (Eng.), Ph.D., and D.Sc. (Eng.) degrees from the University of London, London, U.K., in 1963, 1967, and 1976, respectively.

In 1969, he joined McMaster University, Hamilton, ON, Canada. He is a Professor Emeritus. He was President of Optimization Systems Associates Inc., which he founded in 1983, until November 20, 1997, the date of acquisition by Hewlett-Packard Company. He is President of Bandler Corporation, Dundas, ON, Canada, which he founded in 1997.

Dr. Bandler is Fellow of several societies, including the Royal Society of Canada. He was the recipient of the 2004 IEEE Microwave Theory and Techniques Society (IEEE MTT-S) Microwave Application Award. 